\title{
The up-to-date management of venous thromboembolism 洸滔
}

\author{
Author: Anob M Chakrabarti ${ }^{\mathrm{A}}$
}

There have been a number of developments in the management of venous thromboembolism over the past few years. Old questions, such as thrombolysis, have been revisited in recent trials. New initiatives, such as ambulatory care pathways, are being established across the country. This conference brought together doctors from the UK, USA, Spain and Australia to review the up-to-date management of venous thromboembolism.

KEYWORDS: Venous thromboembolism, pulmonary embolism, thrombolysis, ambulatory care

\section{Prevention of VTE}

The conference was opened by Prof Gerry Stansby from the University of Newcastle, who discussed the vascular surgeon's perspective on venous thromboembolism (VTE) and deep vein thrombosis (DVT). He started with a historical perspective with a quotation from Richard Asher's 1947 article entitled, The dangers of going to bed, where he wrote, 'We may one day regard a thrombosis to be as much a sign of nursing mismanagement as we do the ordinary bed sore today.' While not entirely accurate, today, we do regard hospital-acquired thrombosis as an event needing root cause analysis to ascertain underlying causes, and to allow learning and improvement in policies and processes that are in place.

Prevention remains better than cure. In discussing postoperative DVT he highlighted the importance of appropriate risk assessment in every patient to balance the risk of bleeding (for which surgery is a risk) and of VTE (for which surgery is also a risk). This needs to be considered at an individual level, including both mechanical and/or pharmacological approaches, but also appropriate policies need to be in place for each department. To ensure this is done for every surgical patient, it has been incorporated nationally as part of the World Health Organization surgical safety checklist.

\section{The NICE guidelines and their limits}

The rest of the conference focused predominantly on pulmonary embolism (PE). Accepting that we cannot

Author: Aspecialist registrar in respiratory medicine, Royal Brompton Hospital, London, UK prevent all VTE, Dr Rodney Hughes, from the University of Sheffield, revisited the NICE clinical guideline 144 entitled, Venous thromboembolic diseases: the management of venous thromboembolic diseases and the role of thrombophilia testing. This was issued in June 2012 and deals with diagnosis, treatment and follow up. He reiterated that the diagnosis of PE remains a difficult one and requires good clinical acumen with unexplained acute breathlessness a cardinal symptom. The guidelines cover the familiar diagnostic algorithm using the Wells score, D-dimer and appropriate imaging. Recommended treatment is with low-molecular-weight heparin and inpatient initiation of anticoagulation. As follow up, cancer screening should be offered to patients with unprovoked events over the age of 40 years old.

Since the guidelines were published there have been a number of developments that add more detail and complexity to the management of VTE. These topics formed the core of the rest of the conference. Dr Hughes provided an overview of the latest European Society of Cardiology (ESC) guidelines on the diagnosis and management of acute PE published in $2014^{1}$ which incorporate a number of these new concepts and include:

$>$ risk stratification of patients with $\mathrm{PE}$

$>$ thrombolysis in submassive $\mathrm{PE}$

$>$ the role of new oral anticoagulants (NOACs)

$>$ ambulatory care of patients with $\mathrm{PE}$.

He closed with the important reminder not to lose sight of the patient among all these guidelines and studies, and to address ideas and concerns about the diagnosis and its implications.

\section{Risk stratification of patients with PE}

Prof David Jiménez, from Ramón y Cajal University Hospital in Madrid, reflected on his and others' extensive work on the risk stratification of patients with PE. He opened with the statistic that there is a range of between 0 and $30 \%$ mortality in haemodynamically-stable patients with a PE. ${ }^{2}$ Yet crudely, severity is currently assessed essentially by only one parameter - systolic blood pressure. The importance of being able to subdivide this group and distinguishing low and higher risk normotensive patients was apparent to us all, with its implications for both appropriate medical management and the proper location for their management.

Clinical scores, he argued, provide a cheap, always available tool to enable us to risk stratify. Furthermore he provided evidence from clinical trials showing that direct comparisons favoured clinical scores as compared with, for example, troponin and brain natriuretic peptide in identifying low-risk 
normotensive patients. A number of scoring systems were discussed; the recommendation in the ESC guidelines is to use either the pulmonary embolism severity index (PESI) or the simplified PESI (sPESI). Prof Jiménez advocated use of the sPESI, which he was involved in the development of, on account of its similar prognostic accuracy compared with the original PESI, but greater ease of use in the busy acute medicine setting. ${ }^{3}$

Clinical scores on their own are useful in identifying low-risk patients. However, a different strategy is needed to predict the higher risk normotensive patients more accurately. Interestingly, he defined a submassive PE as a confirmed PE in a normotensive patient, but with an increased mortality similar to patients with cardiovascular instability. This shifts the focus away from right ventricular dysfunction alone, which is the key factor in the ESC definition. He presented data from the PROTECT study which derived and validated a multimarker prognostication model based on sPESI, troponin, brain natriuretic peptide and lower limb ultrasound to arrive at a risk of 30-day mortality and of a complicated clinical course. ${ }^{4}$ The combination of these provides much greater predictive value than each on its own. An online calculator based on this study can be found at www.peprognosis.com. The future in risk stratification now lies in studying whether prognostication per se improves outcomes.

\section{Thrombolysis in submassive PE}

The contentious topic of thrombolysis in submassive PE was the motion for the big debate between two colleagues from the University of Newcastle, with Prof Paul Corris arguing for and Prof John Simpson arguing against. An audience poll prior to the debate had about three-quarters of the audience against. Much of the evidence for this debate focused around the recent PEITHO study ${ }^{5}$ and subsequent meta-analyses. Both sides highlighted the low mortality rate in this group of patients leading to underpowered studies. Prof Corris argued that thrombolysis does result in more rapid and complete resolution of the thrombus with more rapid recovery of right ventricular function. The main battleground was the risk of bleeding and the associated effects. Prof Corris noted that in the PEITHO study, the heparin infusions were titrated to a higher APTT than that recommended by other investigators, and that the optimal dose of thrombolytic agent was not known and would affect the risk-benefit balance. The meta-analyses do suggest that it is possible to identify a subgroup of patients in whom the risk of mortality is high such that it outweighs the risk of bleeding. However, Prof Simpson highlighted that there was an excess of even minor bleeding (requiring transfusion) in the thrombolysis arm. Furthermore, morbidity was not assessed - could the long-term consequences of intracranial haemorrhage be worse than appropriately treated haemodynamic instability? Ultimately while thrombolysis may be warranted on an individual case basis, making it routine was a risky move. He concluded that what was really needed was a trial comparing risk stratification and primary thrombolysis with risk stratification, high dependency monitoring and rescue thrombolysis. The debate ended with the audience voting overwhelmingly against routine thrombolysis in submassive PE.

\section{The role of NOACs}

Dr Trevor Baglin from Addenbrooke's Hospital in Cambridge discussed the role of NOACs, or as he terms them 'nonvitamin K antagonist oral anticoagulants' as they are not really new having been around, albeit not in clinical practice, for the last 10 years. He started by highlighting one of the principles behind the search for NOACs, which was to try to dissociate the antithrombotic from the bleeding effects, to allow effective prevention and treatment of thrombosis without anticoagulantrelated bleeding. The key question is whether they are better, or simply more convenient, that the established therapies. The latter is apparent from a predictable dose response, no need for monitoring and fewer drug-drug interactions. There have been a number of studies demonstrating the non-inferiority of NOACs compared with conventional treatment. Furthermore there are studies both in atrial fibrillation and VTE that have shown a reduced bleeding rate compared with warfarin, particularly with regard to intracranial haemorrhage, suggesting they may be 'better' as well as more convenient. It does seem that the major bleeding with NOACs is mainly from the lower gastrointestinal tract. One of the main concerns is the lack of a reversibility agent, and while the much shorter half-life compared with the effects of warfarin obviates this to some extent, Dr Baglin noted that antidotes were being developed and would likely be available in the next three years. NOACs most definitely have a role in the treatment of patients with acute VTE.

\section{Ambulatory care of patients with PE}

Dr Luke Howard, from Hammersmith Hospital, London is chair of the British Thoracic Society Ambulatory Management of PE Guideline Development Group and offered us his insight into the questions and difficulties that are arising during their discussions. With the political and financial pressure to reduce hospital admissions, it is attractive to try to extend the ambulatory care system for DVT management to PE. The difficulty lies in the fact the PE can be fatal, even in normotensive patients as already described - appropriate patient selection is therefore key. This will likely involve a combination of markers similar to that developed by the PROTECT team and scores such as the Hestia criteria to identify those not safe for ambulatory care.

However, there are a number of important questions to which there is no answer. Can a suspected PE be risk stratified in the same way as a confirmed PE? What does a sPESI score of zero mean in this context? How should cases of PE with a concomitant DVT be managed? None of the NOACs have been trialled in this setting, but will likely have important implications - should we be moving away from low-molecularweight heparin and warfarin?

There are a number of centres across the UK already operating ambulatory PE services with different, locally tailored policies. Judging by the audience response, there will be an increasing number of these services being set up in the next few years and the guidelines will be most timely.

\section{The follow up and complications of VTE}

As Dr Hughes noted earlier, three of the common questions from patients are, 'Why me?', 'Will this happen again?' and 
'What does this mean for my future?' Dr David Keeling from the Oxford University Hospitals tackled the first two questions in his lecture. He argued that what caused the clot was not important beyond classifying events as provoked or unprovoked and excluding cancer, but that the focus of the consultation should be around how long a patient needs to stay on anticoagulation. While three months is necessary for the acute thrombosis, who stops and who carries on involves balancing the risk of recurrence of anticoagulation with the risk of bleeding on warfarin. Unfortunately both of these roughly work out at $0.25 \%$ per annum (although this may change if the risk of bleeding on NOACs is lower). The aim is to try to unbalance this equilibrium by considering factors that either increase the risk of recurrence or the risk of bleeding. Thrombophilia screening rarely influences patient management. Dr Keeling concluded that long-term anticoagulation after a first unprovoked VTE was favoured by male gender, age less than 50 years, post-thrombotic syndrome, $\mathrm{PE}$ and raised $\mathrm{D}$-dimers after completing anticoagulation, but that patient preference was paramount.

Dr David Kiely, from the Royal Hallamshire Hospital, Sheffield, considered the third question, talking about the complications of VTE. In addition to the more widely known entities of post-thrombotic syndrome and chronic thromboembolic pulmonary hypertension (CTEPH), he also discussed the more recently described 'post-PE syndrome' characterised by dyspnoea and decreased exercise capacity, and is on a pre-CTEPH spectrum. ${ }^{6}$ Six months post-PE, $20-70 \%$ of patients report a worse quality of life and $30-50 \%$ are breathless. There are many causes for this, including cardiac dysfunction, obesity and chronic obstructive pulmonary disease. Dr Kiely argued that a PE clinic offering systematic review of patients at the end of their acute anticoagulation phase is an integral part of the PE pathway. This could be a multidisciplinary clinic combining haematology and respiratory to decide both on long-term anticoagulation and also to investigate appropriately persistent breathless patients depending on the likely clinical cause.

\section{Putting together an up-to-date service}

Dr Rodney Jones returned to the lecturn to describe the Sheffield experience and approach to delivering a patientcentred PE service. He highlighted the importance of a multidisciplinary approach and took us through the locally developed PE pathway, building on the latest ESC guidelines to show how they were risk stratifying patients, considering using NOACs and thrombolysis in appropriately selected patients, and delivering an ambulatory care service. This showed us the sort of up-to-date service that could be set up and how its model could be expanded to hospitals across the country by enthusiastic and committed multidisciplinary efforts.

\section{References}

1 Konstantinides SV, Torbicki A, Agnelli G et al. 2014 ESC guidelines on the diagnosis and management of acute pulmonary embolism. Eur Heart J 2014;35:3033-69,3069a-k.

2 Wood KE. Major pulmonary embolism: review of a pathophysiologic approach to the golden hour of hemodynamically significant pulmonary embolism. Chest 2002;121:877-905.

3 Jiménez D, Aujesky D, Moores L et al. Simplification of the pulmonary embolism severity index for prognostication in patients with acute symptomatic pulmonary embolism. Arch Intern Med 2010;170:1383-9.

4 Jiménez D, Kopecna D, Tapson V et al. Derivation and validation of multimarker prognostication for normotensive patients with acute symptomatic pulmonary embolism. Am J Respir Crit Care Med 2014;189:718-26.

5 Meyer G, Vicaut E, Danays T et al. Fibrinolysis for patients with intermediate-risk pulmonary embolism. $N$ Engl J Med 2014;370:1402-11.

6 Klok FA, van der Hulle T, den Exter PL et al. The post-PE syndrome: a new concept for chronic complications of pulmonary embolism. Blood Rev 2014;28:221-6.

Address for correspondence: Dr A Chakrabarti, Royal Brompton Hospital, Sydney Street, London SW3 6NP, UK. Email: a.chakrabarti@rbht.nhs.uk 